\title{
Realismus und unübersetzbare Sprachen
}

„Die Grenzen meiner Sprache bedeuten die Grenzen meiner Welt.. ${ }^{11}$ Mit diesem Zitat beginnt einer der wichtigsten Einwände gegen den Realismus. Wenn die Welt, so der Einwand, nur das ist, was innerhalb der Grenzen meiner Sprache liegt, dann ist das, was jenseits dieser Grenzen liegt, nichts. Daraus folgt, dass alles, was real ist, ausgedrückt werden kann und umgekehrt. Wenn aber Sprache und Realität einander wechselseitig begrenzen, dann verliert die These des Realismus, nach der die Wirklichkeit von uns (und unserer Sprache) unabhängig ist, jeglichen Sinn.

Ich will im Folgenden dieses Argument untersuchen und zeigen, warum ein solcher Einwand zwar scheitert, aber doch eine für den Realismus wichtige Konsequenz enthält. Dazu werde ich in fünf Schritten vorgehen: (1) Ich werde zunächst den Realismusbegriff klären und eine wahrheitstheoretische Definition vorschlagen. Dann werde ich (2) Davidsons Kritik an der Trennung von Begriffsschema und Inhalt als wichtigsten Vertreter des Einwands darstellen. Im nächsten Schritt (3) werde ich einige Gegenargumente zu Davidsons Ansatz diskutieren und anschließend (4) zeigen, weshalb eine unübersetzbare Sprache trotz Davidsons Vorwurf der Inkonsistenz doch möglich ist, um dann auf dieser Basis ein Argument für den Realismus vorzuschlagen. Schließlich werde ich (5) argumentieren, dass eine totale Sprache denkbar ist, deren Möglichkeit den Gegensatz von Realismus und Antirealismus in letzter Konsequenz auflöst.

\section{I}

\section{Realismus}

Realismus ist die These, dass die Wirklichkeit unabhängig von unserem Denken existiert. ${ }^{2}$ Das ist nicht so zu verstehen, dass die Wirklichkeit kein Produkt unseres Denkens ist wie es Träume oder Phantasien sind. Der Realismus stellt vielmehr eine Behauptung über das Verhältnis der Begriffe von Wirklichkeit und Denken auf, nämlich, dass der Begriff der Wirklichkeit unabhängig von mentalen Begriffen verstanden werden muss: Aussagen über die Wirklichkeit implizieren keine Aussagen über mentale Vorkommnisse. ${ }^{3}$ Beispielsweise folgt aus der Behauptung, dass vor dem Haus ein Rosenbusch blüht, nicht, dass aktuell oder potentiell jemand wahrnimmt, dass dort ein Rosenbusch blüht. Entscheidend ist natürlich, was die Unabhängigkeit beider Begriffe bedeutet und aus welchem (materialen, nicht formalen) Grund Sätze über die Wirklichkeit keine Sätze über mentale Vorkommnisse implizieren sollten. Ich werde hier einem Vorschlag Michael Dummetts folgen und

\footnotetext{
${ }^{1}$ Wittgenstein, Tractatus 5.6.

2 Diese Beschreibung ist Konsens sowohl unter Anhängern wie auch Gegnern des Realismus, z.B. Devitt 1991, 14 oder Willaschek 2003, 10

${ }^{3}$ Vgl. Willaschek 2003, 28.
} 
den Realismus als eine semantische These verstehen, d.h. als eine Bedeutungstheorie für eine bestimmte Klasse von Aussagen entweder über die gesamte Realität oder einen ihrer Gegenstandsbereiche, den wir realistisch interpretieren:

The very minimum that realism can be held to involve is that statements in the given class relate to some reality that exists independently of our knowledge of it, in such a way that that reality renders each statement in the class determinately true or false, again independently of whether we know, or are even able to discover, its truth-value. ${ }^{4}$

Der Realismus definiert sich also über den Wahrheitsbegriff, d.h. darüber, was es für die sprachliche Repräsentation eines wirklichen Sachverhalts bedeutet, wahr zu sein. Für den Realismus beziehen sich Aussagen über die Welt auf bestimmte Sachverhalte und sind dementsprechend objektiv wahr oder falsch. Denn wenn die Welt oder ein bestimmter Gegenstandsbereich unabhängig von uns ist, dann liegt die Wahrheit oder Falschheit der Aussagen über diesen Gegenstandsbereich allein in den relevanten Tatsachen, unabhängig von unseren faktischen oder prinzipiellen Erkenntnismöglichkeiten. Daraus ergeben sich zwei Aspekte, die charakteristisch für ein realistisches Verständnis von Wahrheit sind: erstens das Bivalenzprinzip, zweitens die Evidenztranszendenz der Wahrheit. Unter Evidenztranszendenz ist zu verstehen, dass die Wahrheit einer Aussage unsere epistemischen Gründe, sie für wahr zu halten, transzendiert. Das Bestehen aktueller oder potentieller Evidenzen ist für die Wahrheit eines Satzes weder notwendig noch hinreichend Wahrheit ist keine epistemische Eigenschaft, sie kommt einer Proposition allein durch ihre Relation zur Wirklichkeit zu. Daher implizieren Aussagen über die Realität ceteris paribus keine mentalen (epistemisch relevanten) Aussagen. Das Bivalenzprinzip ist die logische Kehrseite der Evidenztranszendenz und besagt, dass jede Aussage (ungeachtet ihres epistemischen Status) genau einen Wahrheitswert hat, wahr oder falsch. Zwar gibt es eine Menge von Aussagen über die Wirklichkeit, die für uns unentscheidbar sind (wir verfügen über keine Belege für oder gegen ihre Wahrheit), aber aus dem Prinzip der Evidenztranszendenz folgt, dass auch diese Sätze definite Wahrheitswerte haben. Das Bivalenzprinzip schließt damit jede Form von Wahrheitswertlücken (truth-value gaps) aus. Realismus bedeutet demnach, dass der Wahrheitswert einer Aussage eindeutig definiert und von epistemischen Faktoren unbeeinflusst ist. Versteht man z.B. Aussagen über die Vergangenheit realistisch, so geht man davon aus, dass diese Aussagen ausnahmslos eindeutig wahr oder falsch sind und dass unsere faktischen oder möglichen Evidenzen für ihre Wahrheit keine Bedingung dieser Wahrheit sind. Damit ergeben sich aus dem Realismus zwei wichtige Konsequenzen: erstens, die Möglichkeit prinzipiell unerkennbarer Tatsachen. Zweitens, die Identität von Wahrheitswerten über alle Sprachen hinweg. Versteht man eine Sprache als die Menge aller Sätze, die in ihr geäußert werden können, so gilt für den Realismus, dass eine Aussage, die in Sprache $L_{1}$ wahr ist, nicht in Sprache $L_{2}$ falsch (oder unbestimmt) sein kann, da dies der Forderung 
des Bivalenzprinzips entgegenlaufen würde, dass jede Aussage einen definiten Wahrheitswert haben muss.

II

\section{Davidsons Argument}

In seinem Text On the very idea of a conceptual scheme legt Davidson das Fundament für eine Kritik des Realismus - auch wenn er selbst das nie explizit sagt. Sein Anliegen ist eigentlich die Auseinandersetzung mit Kuhns und Feyerabends Relativismus, nach dem andere Begriffsschemata neben unserem existieren, die wir nicht teilen und deren Weltbild damit für uns nicht verstehbar ist. Inkommensurable Begriffsschemata enthalten inkompatible Konzepte, die keine Entsprechung im jeweils anderen Schema haben, um die gleichen empirischen Daten zu behandeln. Daraus ergibt sich, dass Theorien aus dem einen Schema nicht in das andere übersetzt werden können, denn die Begriffe, die in Schema A verwendet werden, haben kein Pendant in den Begriffen aus Schema B. Die Inkommensurabilität unterschiedlicher Begriffsschemata impliziert damit die Existenz von unübersetzbaren Sprachen oder zumindest Sätzen. Davidson aber bestreitet, dass die stillschweigend vorausgesetzte Trennung von Begriffsschema und Inhalt sinnvoll ist, und erklärt, es handle sich bloß um ein Dogma. Ist diese Trennung absurd, so muss die Idee, es gäbe einen neutralen Inhalt unseres Denkens, der von unseren jeweiligen Begriffsschemata geformt wird, verworfen werden. Es kann gar keine anderen Begriffsschemata geben, da die Idee eines Begriffsschemas an sich schon inkonsistent ist. Diese Schlussfolgerung hat unmittelbare Auswirkungen auf das Realismusproblem. Wenn wir unter Realismus verstehen, dass die Wirklichkeit nicht von uns abhängt, dann hängt sie auch nicht von unseren Begriffsschemata ab. Der Antirealismus sieht die Welt als bedingt durch unser Begriffsschema, der Realismus bestreitet das. Sollte sich herausstellen, dass man nicht sinnvoll von Begriffsschemata und ihren Inhalten sprechen kann, dann wäre die Konsequenz, dass weder Realismus noch Antirealismus sinnvolle Thesen sind, weil sie auf einer falschen Dichotomie beruhen. Davidsons Kritik basiert auf der These, dass unübersetzbare Sprachen nicht möglich sind, denn da deren Möglichkeit von der Trennung von Schema und Inhalt abhängt, folgt aus ihrer Unmöglichkeit die Falschheit der Trennung:

(1) Wenn es inkompatible Begriffsschemata gibt, dann gibt es Sprachen, die nicht ineinander übersetzbar sind.

(2) Es gibt keine Sprachen, die nicht ineinander übersetzbar sind.

(3) Also gibt es keine inkompatiblen Begriffsschemata.

Formal ist das Argument gültig und der Relativist wird Prämisse (1) sicher zugestehen. Die kritische Frage lautet also, ob Prämisse (2) tatsächlich wahr ist. 
Davidson beginnt seine Begründung mit der Feststellung, dass ein Begriffsschema und eine Sprache immer miteinander verbunden sind: Differenzen in den Begriffsschemata spiegeln sich in den Sprachen wider und sprachliche Unterschiede lassen auf Abweichungen zwischen den Begriffsschemata schließen. ${ }^{5}$ Darauf folgt sein eigentliches Argument: Wenn Sprachen mit Begriffsschemata identifiziert werden können, dann sind inkommensurable Begriffsschemata identisch mit inkommensurablen, also nicht ineinander übersetzbaren Sprachen. Doch dieser Begriff ist nicht konsistent:

Nothing, it may be said, could count as evidence that some form of activity could not be interpreted in our language that was not at the same time evidence that that form of activity was not speech behavior. $^{6}$

Sollten wir also jemals glauben, einen Sprecher einer Sprache vor uns zu haben, die nicht in unsere übersetzbar ist, dann gibt es in Wirklichkeit keinen Grund anzunehmen, dass wir es überhaupt mit einem Sprecher und einer Sprache zu tun haben. Nicht übersetzbare Äußerungen sind nicht unterscheidbar von bloßen Geräuschen. Wir könnten behaupten, das Murmeln der Bäche sei eine Sprache, die wir nicht in die unsere übersetzen können, aber genauso gut könnten wir sagen, es sei gar keine Sprache, sondern nur das bedeutungslose Geräusch fließenden Wassers. Übersetzbarkeit wird damit zu einem Kriterium der Sprachlichkeit. Alles, was Sprache ist, ist auch übersetzbar, oder präziser: Etwas ist eine Sprache genau dann, wenn es in unsere Sprache übersetzbar ist. Das ist äquivalent zur negativen Variante, dass alles, was nicht übersetzt werden kann, auch keine Sprache ist. Damit wäre natürlich die Wahrheit von Prämisse (2) unzweifelhaft erwiesen, denn gemäß diesem Kriterium ist der Begriff einer unübersetzbaren Sprache eine contradictio in adiecto. Aber das ist noch kein Argument, sondern bloß eine andere Formulierung der Prämisse. Aus welchem Grund sollten wir Davidsons Definition von Sprache akzeptieren?

Davidson gibt zu, dass das Kriterium nicht einfach postuliert werden kann, sondern sich als Folgerung aus einem Argument ergeben sollte. ${ }^{7}$ Dazu beruft er sich auf den Zusammenhang zwischen mentalen Zuständen und sprachlichen Äußerungen. Wenn wir die Äußerungen eines Sprechers interpretieren, dann müssen wir ihm zugleich eine Reihe entsprechender mentaler Zustände zuschreiben. Wenn jemand z.B. zu mir sagt: „Heute ist der erste echte Frühlingstag“, dann muss ich, wenn ich seine Äußerung verstehen möchte, annehmen, dass er glaubt, dass heute der erste echte Frühlingstag ist, und werde erwarten, dass er sich so verhält wie jemand, der dies glaubt. Es ist aber, so Davidson, kaum vorstellbar, dass ich in der Lage sein sollte, jemandem derart komplexe mentale Zustände zuzuschreiben, ohne zugleich auch fähig zu sein, diese in Worte zu fassen. ${ }^{8}$ Die Fähigkeit eines Hörers, die Äußerungen eines Sprechers zu interpretieren, setzt voraus, dass der Hörer dem Sprecher

\footnotetext{
${ }_{5}^{5}$ Davidson 1974, 6.

${ }^{6}$ Davidson 1974, 7.

7 Davidson 1974, $7 f$.

8 Davidson 1974, 8.
} 
bestimmte mentale Zustände zuschreibt. Diese Zuschreibung muss in der Sprache des Hörers selbst erfolgen, sonst versteht er seine eigenen Zuschreibungen nicht (oder er muss sie zumindest in seiner Sprache explizieren können). Wenn aber der Hörer die Äußerungen des Sprechers interpretieren kann, dann kann er sie auch übersetzen mit Hilfe der in seiner Sprache ausdrückbaren Zuschreibungen mentaler Zustände. Umgekehrt gilt, dass ein Hörer, der dem Sprecher keine mentalen Zustände zuschreiben kann, ihn auch nicht versteht. Und ein Hörer, der niemals in der Lage ist, dem Sprecher die passenden mentalen Zustände zuzuschreiben (weil seine Sprache nicht in seine eigene übersetzbar ist), wird ihn auch niemals verstehen können. Was aber prinzipiell nicht verstehbar ist, kann keine Sprache sein. Übersetzbarkeit ist also eine notwendige Bedingung für Sprache.

Verfechter des Begriffsrelativismus würden dieses Kriterium jedoch nicht akzeptieren, sondern stattdessen behaupten, dass nicht die Übersetzbarkeit das Kriterium der Sprachlichkeit ist, sondern eine bestimmte Beziehung, die zwischen Sprache und Welt besteht:

The idea is then that something is a language, and associated with a conceptual scheme, whether we can translate it or not, if it stands in a certain relation (predicting, organizing, facing or fitting) to experience (nature, reality, sensory promptings). ${ }^{9}$

Davidson diskutiert zwei mögliche Kriterien, die im Gegensatz zur Übersetzbarkeit eine unübersetzbare Sprache denkbar machen würden: erstens, die Ordnungsfunktion der Sprache, zweitens, die Übereinstimmung von Sprache und Realität. ${ }^{10}$ Beide taugen seiner Meinung nach nicht als ein Kriterium der Sprachlichkeit, das ohne Übersetzbarkeit auskommt.

Zunächst zur Ordnungsfunktion: Sprache kann entweder die Gegenstände der Welt ordnen oder den Strom der Erfahrungen. Dass eine Sprache nicht in eine andere übersetzbar ist, würde dann bedeuten, dass beide Sprachen die Wirklichkeit nach fundamental anderen Prinzipien ordnen. Abweichungen, die sich aus diesen unterschiedlichen Prinzipien ergeben würden, sind aber laut Davidson nur in Einzelfällen erkennbar, und zwar wenn beide Sprachen eine gemeinsame Ontologie voraussetzen. Denn erst die große Zahl der Gemeinsamkeiten lässt die Abweichungen erkennen. Eine Sprache, die einem völlig anderen Ordnungsprinzip folgt, wäre damit zu verschieden, um überhaupt als Sprache erkannt werden zu können. Und umgekehrt gilt, dass eine Sprache, die ähnliche Erfahrungen strukturiert wie die unseren, nicht fundamental anders sein kann als unsere eigene Sprache, und daher prinzipiell übersetzbar sein muss.

Das Argument gegen ein Übereinstimmungskriterium der Sprache ist etwas komplizierter. Zunächst stellt Davidson fest, dass die Idee einer Übereinstimmung zwischen Sprache und Erfahrung dem schlichten Begriff der Wahrheit nichts Nennenswertes hinzufügt. ${ }^{11}$ Man kann zwar sagen, dass die

\footnotetext{
9 Davidson 1974, 13.

10 Davidson 1974, 14.

11 Davidson 1974, 16.
} 
Tatsache, dass Zitronen gelb sind, den Satz „Zitronen sind gelb“ wahr macht, doch diese Aussage behauptet nicht mehr, als dass es wahr ist, dass Zitronen gelb sind. Wenn wir von einem Begriffsschema sagen, es stimme mit der Erfahrung überein, dann meinen wir also eigentlich nur, dass dieses Schema wahr ist. Übertragen auf das Problem der Unübersetzbarkeit bedeutet das: „[Th]e criterion of a conceptual scheme different from our own now becomes: largely true but not

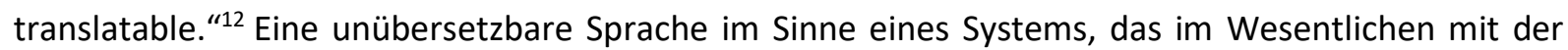
Erfahrung übereinstimmt, wäre also eine Menge von überwiegend wahren Sätzen, die kein Gegenstück in irgendwelchen Sätzen unserer eigenen Sprache haben. Das ist nur dann möglich, wenn der Begriff der Wahrheit unabhängig vom Begriff der Übersetzung verstanden werden kann. Doch gerade das ist laut Davidson unmöglich, denn Tarskis Convention $T$ legt fest, dass für jeden Satz einer Sprache ein T-Satz besteht, der dessen Wahrheitsbedingung angibt. Die Menge aller T-Sätze bestimmt dann die Extension des Wahrheitsbegriffs für die jeweilige Sprache. Davidson räumt ein, dass dies keine Definition des Wahrheitsbegriffs ist, weist jedoch darauf hin, dass Convention $T$ eine Eigenschaft der Wahrheit beschreibt, die jeder Wahrheitsbegriff berücksichtigen muss, nämlich die Übersetzbarkeit des objektsprachlichen Satzes in eine metasprachliche Angabe seiner Wahrheitsbedingung. ${ }^{13}$ Kein Wahrheitsbegriff kann weniger sein als das (oder etwas komplett Anderes), so dass Übersetzbarkeit eine notwendige Bedingung von Wahrheit ist. Das vermeintliche Alternativkriterium der Sprachlichkeit impliziert damit einen Widerspruch - nichts kann zugleich wahr und doch unübersetzbar sein, denn der Wahrheitsbegriff ist nicht definiert über die Menge übersetzbarer Sätze hinaus. Zusammengefasst:

(a) Der Begriff der Bedeutung schließt unübersetzbare Sprachen aus. Etwas, das Bedeutung hat, ist eine sprachliche Äußerung. Wenn eine bestimmte Form von Verhalten als sprachliche Äußerung interpretiert werden soll, dann müssen wir voraussetzen, dass das Wesen, das dieses Verhalten zeigt, bestimmte mentale Zustände besitzt. Wenn diese mentalen Zustände für uns erkennbar sind, dann können sie systematisch mit dem mutmaßlichen Sprachverhalten in Beziehung gesetzt werden, so dass sie die Brücke bilden, um die Sprache zu übersetzen. Können Sie nicht in Beziehung gesetzt werden, gibt es keine Übersetzung, aber ebenso keine Bedeutung, also auch keine Sprache.

(b) Der Begriff der Wahrheit schließt unübersetzbare Sprachen aus. Der Begriff der Wahrheit ist definiert durch seine Extension in einer Sprache, d.h. durch die Liste aller T-Sätze, in denen die Wahrheitsbedingungen jedes objektsprachlichen Satzes in einer Metasprache genannt werden. Ein Satz, für den wir keine Wahrheitsbedingung angeben können, ist also zugleich ein Satz, der nicht übersetzt werden kann, denn Übersetzung impliziert, dass zwei Sätze in zwei unterschiedlichen Sprachen die gleiche Bedeutung und damit die gleiche Wahrheitsbedingung haben. Kann ein Satz

\footnotetext{
12 Ebd.

${ }^{13}$ Davidson 1974, 17.
} 
nicht übersetzt werden, so kann er also weder wahr sein noch Bedeutung tragen, und ist daher im eigentlichen Sinne kein Satz. Wahrheit ist eine notwendige Bedingung für Sprachlichkeit und Übersetzbarkeit eine Bedingung für Wahrheit.

Was folgt hieraus für den Realismus? Oberflächlich betrachtet müssten wir eigentlich den Realismus ebenso wie seinen Widerpart aufgeben, da beide auf der unhaltbaren Voraussetzung beruhen, dass es ein Begriffsschema geben könnte, von dem die Welt abhängig ist oder auch nicht. Aber so einfach ist es doch nicht. Zwar dürften wir laut Davidson eigentlich nicht sagen, dass unser Begriffsschema bestimmt, was für uns die Welt ist, dass also die Grenzen meiner Sprache die Grenzen meiner Welt sind - aber natürlich ist es genau das, was er eigentlich behauptet. Die Vorstellung einer Sprache als Begriffsschema, das einen Inhalt ordnet, mag laut Davidson absurd sein, aber am Ende läuft Davidsons Gedankengang doch darauf hinaus, dass die einzige Wirklichkeit, die es für uns geben kann, die ist, die sich sprachlich erfassen lässt. Denn indem sowohl Wahrheit als auch Bedeutung an die Bedingung der Übersetzbarkeit geknüpft werden, wird jede Möglichkeit einer Wahrheit, die nicht in unsere Sprache übersetzt werden kann, ausgeschlossen. Die Grenzen meiner Sprache sind eben doch die Grenzen meiner Welt, denn meine Sprache begrenzt alles, was überhaupt wahr und sinnvoll sein kann. Nur dass Davidson gerne das Wort ,Grenzen' vermeiden möchte, denn eine Grenze ist etwas, das zwei Dinge voneinander trennt. Wo es aber nichts zu trennen gibt, braucht es auch keine Grenze. Ob nun aber die Grenzen meiner Sprache die Grenzen meiner Welt sind oder meine Sprache meine Welt ist, ist bloß Haarspalterei. Davidsons vermeintliche Überwindung des Gegensatzes von Realismus und Antirealismus entpuppt sich damit als Verteidigung eben jenes Antirealismus.

III

\section{Einwände}

Akzeptieren wir einmal Davidsons Begründung seines Kriteriums der Übersetzbarkeit. Dann stellt sich dennoch die Frage: Warum ist gerade unsere Sprache so mächtig, dass sie alles ausdrücken kann? Thomas Nagel entwirft ein Szenario, das die Plausibilität des Übersetzbarkeitskriteriums untergraben soll: ${ }^{14}$ Wir wissen aus eigener Erfahrung, dass es Wesen gibt, deren kognitive Fähigkeiten nicht an unsere eigenen heranreichen. Ein Kind von neun Jahren ist beispielsweise nicht dazu in der Lage, die Allgemeine Relativitätstheorie zu verstehen, weshalb bestimmte Aussagen, die im Rahmen dieser Theorie über Raum und Zeit gemacht werden können, nicht in die Sprache des Neunjährigen übersetzbar sind. Nun werden Neunjährige mit der Zeit älter und lernen dazu, so dass ein Dreißigjähriger vielleicht in der Lage ist, zu verstehen, was der Neunjährige nicht verstehen konnte. Aber wir haben keine Schwierigkeiten, uns eine Spezies von Lebewesen vorzustellen, deren kognitive

\footnotetext{
${ }^{14}$ Vgl. Nagel 1986, 95ff.
} 
Fähigkeiten niemals über das Niveau neunjähriger Menschen hinausgehen. Nehmen wir an, es gäbe eine solche Spezies, und diese Spezies hätte ihren eigenen Davidson hervorgebracht, der die These vertritt, dass nur das als Sprache anerkannt werden kann, was prinzipiell in seine Sprache übersetzbar ist. Dann ist aus unserer Perspektive völlig klar, dass der neunjährige Davidson sich irrt. Auch wenn die Aussagen der Allgemeinen Relativitätstheorie über Raum und Zeit nicht in seine Sprache übersetzbar sind, wissen wir aufgrund unserer höheren kognitiven Fähigkeiten genau, dass es solche Aussagen gibt, welche Bedeutung sie haben und dass sie wahr sind. Unsere Sprache enthält demnach Sätze, die nicht in die Sprache der Neunjährigen übersetzbar sind - doch wir würden niemals auf die Idee kommen, diese Sätze deshalb für sinnlos oder unverständlich zu halten. Wenn aber diese Situation, in der wir der überlegene Part sind, problemlos vorstellbar ist, warum sollte es nicht auch denkbar sein, dass es andere Wesen gibt, die zu uns im gleichen Verhältnis stehen wie wir zu den hypothetischen Neunjährigen? Das Urteil dieser Wesen über unseren Davidson müsste dann genau so ausfallen wie unseres über den der Neunjährigen. Der einzige Weg, diese Konsequenz zu vermeiden, bestünde darin, anzunehmen, dass wir uns an einer besonderen Stelle des Spektrums kognitiver Fähigkeiten befinden, nämlich an seinem Ende. Nur wenn es keine kognitiven Fähigkeiten gibt, die über unsere hinausgehen, ist die Annahme derart überlegener Wesen unmöglich, und wir könnten weiterhin mit Davidson daran festhalten, dass alles in unsere Sprache übersetzbar sein muss. Aber welchen Grund haben wir, anzunehmen, dass gerade wir diese herausragende und höchst unwahrscheinliche Position einnehmen? Ohne gute Gründe dafür ist die einzig rationale Position ein "epistemischer Kopernikanismus": Wir haben keinen Grund zu der narzisstischen Annahme, dass wir in irgendeiner Weise einen besonderen Platz im Spektrum kognitiver Fähigkeiten einnehmen. ${ }^{15}$ Dann gibt es aber auch keinen Grund zu glauben, gerade in unserer Sprache sei das Maximum an Ausdrucksfähigkeit erreicht.

Damit ist Davidsons Argument noch nicht erledigt - es könnte ja immer noch Gründe geben, die dafür sprechen, uns den ersten Platz im Ranking der kognitiven Kapazitäten zuzusprechen. Aber Nagels Gedankenexperiment sollte uns das Argument suspekt machen, denn wenn Davidson Recht hat, folgen daraus Konsequenzen, die so unwahrscheinlich sind, dass sie unter anderen Umständen ohne Zögern ausreichen würden, das Argument zu verwerfen. Ein Argument, das nachweist, dass die Erde der Mittelpunkt des Universums oder dass der menschliche Körper die vollkommenste aller physischen Gestalten ist, würden wir allein aufgrund dieser ebenso egozentrischen wie unwahrscheinlichen Konsequenz anzweifeln. Aber wo liegt der Fehler, außer in der protagoreischen Hybris, den Menschen als Maß aller Dinge zu nehmen? Im Übersetzbarkeitskriterium. Denn erstens ist schon der Begriff der Übersetzung unklar, und zweitens lassen sich klare Beispiele gegen das Kriterium finden.

\footnotetext{
${ }^{15} \mathrm{Vgl}$. Rescher 1980, 335.
} 
Für Davidson gilt ein Satz dann als Übersetzung eines anderen Satzes, wenn beide die gleichen Wahrheitsbedingungen haben. Exakte Gleichheit der Wahrheitsbedingungen ist aber in vielen Fällen gar nicht ohne weiteres möglich oder nicht klar erkennbar. Man kann z.B. den englischen Satz "I just had dinner with a friend“ übersetzen mit "Ich habe nur mit einem Freund zu Abend gegessen" oder mit „Ich habe nur mit einer Freundin zu Abend gegessen“; es kommt auf den Kontext an, um zu entscheiden, welche der Bedeutungen gemeint ist. Ohne Kontext sind die Wahrheitsbedingungen beider deutschen Sätze die gleichen wie die des englischen Satzes, so dass eigentlich beide als richtige Übersetzungen gelten müssten. Ist der englische Satz dann überhaupt ins Deutsche übersetzbar? Die Antwort ist nicht klar, ohne dass wir deshalb Zweifel an der Sprachlichkeit des Englischen hätten.

Man könnte einwenden, dass es sich hier bloß um ein technisches Problem handelt, das durch Präzisierung des Übersetzungsbegriffs gelöst werden könnte. Doch selbst wenn wir dies akzeptieren, bleibt noch ein weiteres Problem, nämlich dass Fälle existieren, die offensichtlich gegen das Kriterium sprechen: ${ }^{16}$ Das Etruskische kann bisher nur sehr dürftig übersetzt werden, das Minoische, das uns in Linear-A-Texten überliefert ist, gar nicht. Trotzdem gibt es keinen Grund, daran zu zweifeln, dass es sich hier um Sprachen handelt. Rein syntaktische oder kontextuelle Fakten reichen aus, um diesen Schluss zu ziehen: die Form und Anzahl der gebrauchten Zeichen, ihre statistische Häufigkeitsverteilung und Entropie, die Verwendung in bestimmten Kontexten wie Handel, Kommunikation oder Verwaltung - all das ist Grund genug, bestimmte Zeichen für sprachlich zu halten, auch wenn wir nicht die geringste Ahnung davon haben, was sie bedeuten. Übersetzbarkeit ist also nicht notwendig für Sprachlichkeit.

Aber, so wird man einwenden, Davidson geht es gar nicht um die faktische Möglichkeit zur Übersetzung. Sicher, in manchen Fällen sind bestimmte Sprachen für uns aus kontingenten Gründen unübersetzbar, z.B. weil uns ein Stein von Rosetta fehlt, d.h. ein Schlüssel, um in den Code einzudringen. Dann aber ist nicht die Sprache unübersetzbar, sondern wir sind nicht in der Lage sie zu übersetzen. Unter günstigeren Umständen wäre die Übersetzung prinzipiell möglich. Im Gegensatz dazu bestreitet Davidson die Möglichkeit einer Sprache, die notwendigerweise unübersetzbar ist, für die es also aus begrifflichen Gründen niemals einen Stein von Rosetta geben kann. Dann sollten wir allerdings Davidsons These etwas anders verstehen: Was Davidson im Sinn hat, ist eigentlich gar nicht Übersetzbarkeit, sondern Interpretierbarkeit. ${ }^{17}$ Ein Begriffsschema ist dann radikal von unserem verschieden, wenn wir nicht in der Lage sind, bestimmte Handlungen eines anderen Wesens als Sprache zu interpretieren, unabhängig davon, ob wir in irgendeiner Weise fähig sind, diese Sprache de facto zu übersetzen.

\footnotetext{
${ }^{16}$ Rescher 1980, 327; Sankey 1990, 8f.

17 So auch Rescher 1980, 327.
} 


\section{Unverstehbare Sprachen}

Gibt es Fälle, die gegen dieses modifizierte Kriterium sprechen, d.h. gibt es Formen des Verhaltens, bei denen es sich um sprachliche Äußerungen handelt, die wir aus welchen Gründen auch immer nicht verstehen oder als solche erkennen können? Es gibt alltägliche Situationen, in denen wir etwas als Sprache identifizieren, aber nicht verstehen können: Wenn wir z.B. in der U-Bahn Menschen hören, die sich in einer fremden Sprache unterhalten, erkennen wir, dass es sich um eine Sprache handelt, aber wir verstehen sie nicht. Das ist allerdings kein Einwand gegen Davidson, denn wir könnten die Sprache ja lernen - die Sprache ist nicht an sich uninterpretierbar, sie ist es nur zufälligerweise für uns. Ähnlich ist es, wenn wir in einer Sprache, die wir eigentlich sprechen, auf einen Begriff stoßen, den wir nicht verstehen (auch fortgeschrittene Lerner des Deutschen werden vielleicht irritiert gucken, wenn man ihnen sagt, sie sollen keine Fisimatenten machen). Man versteht zwar die Äußerung kontingenterweise nicht, kann aber lernen, sie zu verstehen. Doch es gibt auch Fälle, in denen das Lernen schwieriger fällt, und in denen wir einen Begriff nicht einfach übersetzen können, etwa weil uns das entsprechende Konzept fehlt. Das ist bei Begriffen der Fall, die in unserer Sprache keine Entsprechung haben, da sie stark an bestimmte kulturelle Kontexte gebunden sind wie z.B. das chinesische 气 (Qi). Aussagen über Qi lassen sich nicht ohne weiteres verstehen; wir müssen erst das Konzept Qi begreifen, seine Verwendungsregeln und seine Einbindung in sprachliche und kulturelle Praktiken erfassen. Erst wenn die kulturelle Distanz überwunden ist und wir uns das Konzept angeeignet haben, verstehen wir den Begriff. Doch auch das ist noch kein Fall von Unverstehbarkeit. Einfaches Nachschlagen im Wörterbuch mag nicht ausreichen, um zu verstehen, was Qi bedeutet, aber eine langwierige und gründliche Einübung in die entsprechende kulturelle Praxis wird schließlich zum Verstehen des Begriffs führen. Ist ein Begriff denkbar, der nicht verstehbar und auch nicht erlernbar ist? Eine derart radikale Unverständlichkeit ist nur vorstellbar unter der Bedingung, dass zum Verstehen der Äußerung das Erfassen von Konzepten notwendig ist, die uns nicht zugänglich sind und auch nicht sein können. Es muss sich also um Äußerungen handeln, deren Inhalt die Kapazitäten unseres Geistes übersteigt. Sollte eine solche Sprache vorstellbar sein, so würde das tatsächlich Davidsons Kriterium widerlegen.

Allerdings hatte Davidson genau diese Vorstellung als absurd zurückgewiesen und erklärt, dass nichts als Indiz dafür gelten kann, dass wir es mit einer unverstehbaren Sprache zu tun haben, was nicht zugleich ein Indiz dafür ist, dass es sich gar nicht erst um eine Sprache handelt. ${ }^{18}$ Wie kann eine Sprache Begriffe enthalten, die prinzipiell nicht verstehbar und erlernbar sind - was könnte es

\footnotetext{
18 Davidson 1974, 7.
} 
rechtfertigen, hier noch von Begriffen zu sprechen? Wir benötigen ein alternatives Kriterium der Sprachlichkeit, das ohne Bezug auf Verstehen auskommt. In seiner Erwiderung auf Davidson schlägt Rescher vor, die funktionale Rolle der Sprache (und nicht ihre Versteh- oder Übersetzbarkeit) als wesentliches Merkmal anzusehen:

What makes such processes into uses of a language is their function in communicative transactions in transmitting information, coordinating action, eliciting responses, and the like. Not translatability as such, but functional equivalency is the determinant of language use. ${ }^{19}$

Etwas ist demnach eine Sprache, wenn es der Kommunikation bzw. Organisation und Steuerung von Verhalten dient. Sprache ist all das, was die Kommunikationsrolle erfüllt. Das funktionale Verständnis von Sprache macht verständlich, weshalb entgegen Davidsons Behauptung eine unverstehbare Sprache doch denkbar ist: Eine unverstehbare Sprache ist Kommunikation, die nicht als solche erkennbar ist. Dass Kommunikation stattfindet, ist unabhängig davon, ob ein externer Beobachter in der Lage ist, diese Kommunikation auch als solche zu erkennen. Ob und unter welchen Bedingungen wir einen bestimmten Prozess oder ein Ereignis als Kommunikation bezeichnen, hängt von Faktoren $a b$, die über die objektiv beobachtbaren Fakten hinausgehen. Kommunikation setzt zwar in objektiver Hinsicht die (physikalische) Übertragung von Information voraus, ist aber nicht identisch mit ihr. So können scheinbar zufällige und bedeutungslose Ereignisse, wenn sie einem vorher festgelegten Code folgen, ein Mittel der Kommunikation sein, das von außen nicht als solches erkennbar ist, weil für den Beobachter nicht nachvollziehbar ist, ob sie eine funktionale Rolle spielen (oder falls ja, welche).

Wenn aber Kommunikation nicht notwendigerweise von außen identifizierbar ist, wie kann dann das entsprechende Verhalten als Sprachverhalten erkannt werden? Putnam schließt das kategorisch aus: "[l]f we cannot interpret organisms's noises at all, then we have no ground for regarding them as thinkers, speakers, or even persons. ${ }^{120}$ Doch diese Annahme geht zu weit. Es ist nicht notwendig, eine Sprache auch zu verstehen, um sie als Sprache zu identifizieren - rein kontextuelle Faktoren können dazu bereits hinreichen: „Travellers recognise native speech as the local tongue without understanding it. ${ }^{\prime 21}$ Diese kontextbasierte Identifizierbarkeit beruht nun ihrerseits allerdings auf der kritischen Voraussetzung, dass wir in der Lage sind, die Kontexte als Sprachkontexte zu erkennen. Denn wenn wir die relevanten Kontexte verstehen, d.h. in der Lage sind, die Handlungen und Intentionen der Akteure zu erkennen (z.B. im Café etwas bestellen, eine Freundin begrüßen, einen Brief schreiben), dann - so könnte Putnam einwenden - können wir ja prinzipiell wieder die Sprache interpretieren. Vielleicht ist nicht jede sprachliche Äußerung für uns exakt übersetzbar, aber wir

\footnotetext{
19 Rescher 1980, 328.

20 Putnam 1981, 114.

${ }^{21}$ Sankey 1990, 9.
} 
können uns über den Kontext ihrer Bedeutung annähern: In dem Moment, in dem wir ein Ereignis, in das zwei Individuen involviert sind, als ,A begrüßt $B^{\prime}$ erkennen, haben wir einen Weg gefunden, die Aspekte des Ereignisses, bei denen es sich um Kandidaten für sprachliche Äußerungen handelt, zu interpretieren. ${ }^{22} \mathrm{Um}$ eine Sprache kontextuell als Sprache zu identifizieren, muss demnach der Kontext anderen, uns vertrauten Kontexten hinreichend ähnlich sein.

Je ferner und fremder uns ein Wesen oder eine Lebensform ist, umso schwerer fällt es uns, Kontexte der Sprachverwendung und damit die funktionalen Rollen mutmaßlicher sprachlicher Äußerungen zu identifizieren. Ein Beispiel, das bizarrer ist als der Unterschied zwischen Naturwissenschaft im 18. und im 20. Jahrhundert, den Davidson und Putnam als Paradigma nehmen, mag dies illustrieren. In Olaf Stapledons Roman Star Maker, einer skurrilen Verschmelzung von Science-Fiction und Neuplatonismus, gelangt der Erzähler im Lauf seines kosmischen Aufstiegs zum Einen zu der Erkenntnis, das auch die Sterne Lebewesen sind, die über eine eigenartige Form von Bewusstsein verfügen, und deren scheinbar durch die Gesetze der Gravitation bestimmte Bewegungen in Wahrheit bewusste und willentliche Handlungen sind. In einem solchen Szenario stellt sich die Frage, ob und wie man überhaupt zu dieser Einsicht gelangen könnte. Wie kann entschieden werden, welche physischen Ereignisse Kandidaten für Interpretationsversuche sind, was also überhaupt in Frage kommt als möglicher Träger der funktionalen Rolle der Sprache? Bei Menschen einer fremden Kultur mag das einfach sein - es sind die Laute, die sie produzieren. Wir wissen ja, dass menschliche Sprache durch Schallwellen funktioniert, die unsere Sprechorgane produzieren. Wie aber ließe sich bestimmen, in welchem Medium eine vollkommen fremde Lebensform kommuniziert? Signale sind Träger einer Information, aber wenn wir einen rein statistischen Begriff von Information annehmen, dann gilt, dass jedes physische Ereignis Information trägt und ein Signal ist. Zur Kommunikation wird es aber erst dann, wenn wir es als Träger der Sprachrolle identifizieren und interpretieren, was wieder von unserer Fähigkeit abhängt, den relevanten Kontext zu erkennen.

Die Zuschreibung einer funktionalen Rolle ist also selbst voraussetzungsbeladen, denn wenn wir ein Signal kontextbasiert als sprachliche Äußerung identifizieren, machen wir damit bereits bestimmte Annahmen über den Verursacher des Signals (den wir in diesem Fall als Sprecher bezeichnen können), insbesondere, dass wir es mit einer bewussten, handelnden Person zu tun haben. Wenn die Blätter eines Baumes im Wind rauschen, so ist das ein physikalisches, rein kausal erklärbares Ereignis. Wenn jemand mich fragt, wo der Bahnhof ist, so reicht eine rein physikalische Beschreibung nicht mehr zur Erklärung dieses Ereignisses aus; ich muss Bezug nehmen auf die intentionalen Zustände des Anderen, seinen Wunsch, mit dem Zug zu fahren, sein Unwissen bezüglich der Lage des Bahnhofs etc., was nichts anderes bedeutet, als dass ich ihn (im Gegensatz zum Baum) als Person verstehen muss (dies ist notwendigerweise Teil des Kontextes, der die Interpretation ermöglicht). Die Fähigkeit zur

22 Das entspricht dem Vorgehen, das Davidson in Radical Interpretation beschreibt. 
Sprache ist jedoch eine notwendige Bedingung der Personalität, ${ }^{23}$ womit die Sache zirkulär wird. Etwas als Sprache zu identifizieren heißt, den Sprecher als Person anzuerkennen, und den Sprecher als Person anzuerkennen, heißt, ihm unter anderem Sprachfähigkeit zuzuschreiben. Wenn wir jemanden/etwas als handelnde Person beschreiben, nehmen wir an, dass er über Bewusstsein und Sprache verfügt, und dass er über Bewusstsein und Sprache verfügt, ergibt sich aus der Interpretation seiner Handlungen. Verstehen wir jemanden/etwas nicht mehr als Handelnden, ist es nicht mehr möglich, irgendwelche physischen Ereignisse, in die er/es verwickelt ist, als Handlungen und damit als Sprachverhalten zu deuten. Dennett betont, dass ein weiteres wichtiges Kriterium der Personalität darin liegt, von anderen als Person wahrgenommen und behandelt zu werden, ${ }^{24}$ so dass der Begriff der Person unvermeidlich zu einem normativen Begriff wird. ${ }^{25}$ Wenn wir etwas als Person, als Sprache oder als Handlung bezeichnen, handelt es sich nicht um eine Beschreibung physischer Fakten, sondern um eine evaluative Einschätzung solcher Fakten, die (wollen wir nicht in einen naturalistischen Fehlschluss geraten) niemals durch die Fakten allein begründet werden kann. Die Kontexte, auf denen unsere Zuschreibung einer funktionalen Rolle basiert, sind damit irreduzibel normativ.

Das Problem daran ist, dass unsere Bewertung von Dingen als Personen offensichtlich abhängig ist von ihrer Ähnlichkeit mit uns selbst. ${ }^{26}$ Wir betrachten Dinge als Personen, wenn sie Bewusstsein und Sprachfähigkeit besitzen, und die Kriterien für das Vorhandensein dieser beiden Faktoren entnehmen wir unserer eigenen Erfahrung. Bewegen wir uns (wie Davidson offenbar stillschweigend voraussetzt) nur im Bereich des Menschlichen, zwischen verschiedenen Kulturen und Epochen, so ist die Fremdheit nicht ausgeprägt genug, um ernsthafte Konfliktfälle auftreten zu lassen. Doch je fremder uns eine Lebensform ist, umso größer werden unsere Probleme, überhaupt eine Form von Verhalten auszumachen, die wir als Sprache deuten können. Ist ein Wesen uns sehr unähnlich (man denke etwa an den lebendigen Ozean in Stanislaw Lems Solaris und die immer wieder scheiternden Versuche, die physischen Gebilde, die er produziert, als Kommunikation zu interpretieren), etwa weil seine Existenzweise vollkommen anders ist als unsere, so wird dieses Wesen über gänzlich andere Begriffe verfügen, um seine Bewusstseinsinhalte zu strukturieren, und es wird in seinen Handlungen auf völlig

\footnotetext{
${ }^{23}$ So etwa Dennett 1981, 270.

${ }^{24}$ Ebd.

25 Dennett 1981, 285.

${ }^{26}$ Diesen Punkt hat besonders Husserl in seinen Cartesianischen Meditationen herausgearbeitet. Die Konstitution eines Wesens als Anderer, d.h. als bewusstes, personales Wesen wie ich selbst, geht von unserer eigenen Selbsterfahrung aus. Der andere ist buchstäblich ein alter ego, ein anderes Ich, das nur als solches erkennbar ist aufgrund seiner Ähnlichkeit zu uns selbst, die es erlaubt, eine Analogie zum eigenen Erleben zu ziehen: „Nehmen wir an, es tritt ein anderer Mensch in unseren Wahrnehmungsbereich, so heißt das primordinal reduziert: es tritt im Wahrnehmungsbereich meiner primordinalen Natur ein Körper auf, der als primordinaler natürlich bloß Bestimmungsstück meiner selbst [...] ist. Da in dieser Natur und Welt mein Leib der einzige Körper ist, der als Leib [...] ursprünglich konstituiert ist [...], so muß der Körper dort, der als Leib aufgefaßt ist, diesen Sinn von einer apperzeptiven Übertragung von meinem Leib her haben [...]. Es ist von vornherein klar, daß nur eine innerhalb meiner Primordinalsphäre jenen Körper dort mit meinem Körper verbindende Ähnlichkeit das Motivationsfundament für die analogisierende Auffassung des ersteren als anderer Leib abgeben kann.“ (Hua 1/140)
} 
andere Art mit seiner Umwelt interagieren als wir. Einem solchen Wesen würden wir vermutlich niemals Sprachlichkeit zuschreiben, weil wir nicht in der Lage sind, von inm verursachte Ereignisse als Sprache zu interpretieren.

Dann ist aber auch klar, warum Davidson glaubt, dass es keine unverstehbare Sprache geben kann: Sprache lässt sich nur zuschreiben aufgrund von kontextuell gegebenen Kriterien wie Personalität oder Intentionalität, die einerseits selbst wiederum Sprache voraussetzen, die wir aber andererseits nur dann sinnvoll zuschreiben können, wenn die relevanten Subjekte uns hinreichend ähnlich sind. Daraus folgt, dass, wenn wir etwas als Sprache bezeichnen, bereits klar ist, dass die Sprecher uns prinzipiell vergleichbare, bewusste und personale Wesen sind, so dass immer die Möglichkeit besteht, einen Weg zu finden, ihre Äußerungen zu interpretieren. Umgekehrt werden wir all das, was aufgrund massiver Differenzen zu uns selbst für uns de facto unverstehbar ist, nicht als Sprache bezeichnen. Unverstehbare Sprachen sind nicht unmöglich, sondern für uns nicht als Sprachen erkennbar. Wir betrachten Chinesisch als Sprache, weil wir Chinesen als Personen wahrnehmen, und wir nehmen sie als Personen wahr, weil sie in ihren Handlungen Intentionalität und rationales Verhalten zeigen, wie wir es aus unserem eigenen Erleben kennen. Betrachten wir etwas nicht als Sprache, dann deshalb, weil wir es keinem personalen Wesen zuordnen können, und dies deshalb nicht, weil wir keine Handlungen und damit keine Intentionalität und Rationalität ausmachen können, die uns das Wesen verstehbar machen würden. Die Bedingungen der Zuschreibung von Sprache stellen bereits sicher, dass wir nur solche Phänomene als Sprache betrachten, die wir auch prinzipiell verstehen können, da wir einen Kontext, in dem wir keinem der Beteiligten personale Qualitäten zuschreiben, gar nicht als Sprachkontext erkennen würden. Das heißt aber nicht, dass andere Phänomene nicht ebenso Sprache sein können; Davidson verwechselt hier ein epistemisches Kriterium mit einem ontologischen. Unverstehbare Sprachen sind möglich, aber unerkennbar.

Nun könnte man in Davidsons Sinn immer noch Folgendes einwenden: Wenn unverstehbare Sprachen nicht als solche erkennbar sind, wie könnten wir dann jemals Grund haben, etwas als unverstehbare Sprache anzusehen? Selbst wenn die Existenz unverstehbarer Sprachen möglich ist, welchen Grund haben wir zu der Annahme, es könnte sie de facto geben? Darauf sind zwei Antworten möglich. Erstens: Wir müssen unterscheiden zwischen einer vollständig und einer partiell unverstehbaren Sprache. Eine vollständig unverstehbare Sprache ist eine, die mit unserer eigenen Sprache keinerlei Begriffe teilt, und die demnach auch für uns niemals als Kommunikation erkennbar ist, weil es keinen Anknüpfungspunkt für eine Analogisierung der Verhaltenskontexte geben kann. Eine partiell unverstehbare Sprache teilt eine gewisse Menge von Begriffen mit unserer Sprache, enthält aber auch eine Menge von Begriffen, die keine Entsprechung in unserer Sprache haben und kann daher anhand des geteilten begrifflichen Repertoires immer noch als Sprache erkannt werden. Die Sprachen zweier voneinander verschiedener menschlicher Kulturen sind partiell unverstehbare 
Sprachen, etwa homerisches Griechisch und modernes Deutsch: Unsere psychologischen Vorstellungen unterscheiden sich so stark von denen des archaischen Griechenland, dass wir die

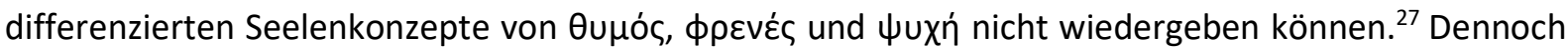
teilen sich beide Sprachen hinreichend viele Begriffe und sind sich in ihren Gebrauchskontexten hinreichend ähnlich, um wechselseitig als Sprache erkennbar zu sein. Aus der Existenz solcher partiell unverstehbarer Sprachen lässt sich die Möglichkeit vollständig unverstehbarer Sprachen extrapolieren, da der Unterschied zwischen beiden nur graduell ist. Zweitens: Es ist eine Konsequenz des eben genannten kopernikanischen Arguments von Nagel und Rescher, dass wir höchstwahrscheinlich epistemisch begrenzt sind, d.h. unfähig, alle logisch möglichen Begriffe zu bilden. Dann aber ist auch die Existenz unverstehbarer Sprachen wahrscheinlich. Denn wenn wir nicht alle Begriffe bilden können, muss es Begriffe geben, über die wir nicht verfügen und die Wesen mit einer mentalen Struktur wie der unseren nicht lernen können, woraus folgt, dass es Propositionen geben kann, in denen diese Begriffe vorkommen, und die für uns nicht verstehbar sind. Alle Sprachen, die diese Propositionen ausdrücken können, sind für uns dann (partiell) unverstehbar. Zwar folgt die Existenz solcher Sprachen nicht mit Notwendigkeit aus der Tatsache unserer epistemischen Begrenzung, sondern nur ihre Möglichkeit. Aber: Fügen wir das erwähnte kopernikanische Argument hinzu, so ist es unwahrscheinlich, dass keine unverstehbaren Sprachen existieren. Denn es ist zwar möglich, dass alle existierenden Sprachen in unsere Sprache übersetzbar sind, wenn nämlich alle theoretisch denkbaren umfassenderen Sprachen nicht realisiert sind (und wir tatsächlich die Spezies mit den höchsten kognitiven und kommunikativen Kapazitäten im Universum sind), aber wahrscheinlich ist es nicht. Wenn unendlich viele komplexere Sprachen denkbar sind, welchen Grund gibt es dann anzunehmen, dass ausgerechnet menschliche Sprachen sich in der besonderen Position befinden, die komplexesten zu sein (selbst wenn es mit Davidson keine direkten empirischen Belege dagegen geben kann)?

\section{Realismus und Antirealismus: Ein Berkeley'sches Argument}

Davidsons Übersetzbarkeitskriterium ist falsch. Wahrheit und Bedeutung sind nicht auf den Bereich unserer Sprache beschränkt, denn eine nicht interpretierbare Sprache ist denkbar (damit fällt auch Davidsons Argument für die Aufhebung der Schema-Inhalt-Distinktion weg). Was folgt daraus für das Realismusproblem?

\footnotetext{
${ }^{27}$ Genaugenommen ist es schon falsch, hier überhaupt von Seelenkonzepten zu sprechen, da das bereits die Annahme suggeriert, es gäbe einen Oberbegriff, unter den die drei untergeordnet werden könnten. Doch einen solchen Begriff kennt das homerische Griechisch nicht - die drei Begriffe stehen nebeneinander und werden erst als Arten einer Gattung angesehen, wenn es bereits das Konzept einer einheitlichen Seele gibt. Ausführlich dazu siehe Snell 1975, Kap. I.
} 
Davidsons These, dass alles, was eine Sprache ist, verstehbar und damit auch in unsere Sprache übersetzbar sein muss, impliziert, dass unsere Sprache alles ausdrücken kann, was überhaupt ausdrückbar ist. Nun ist alles, was ausgedrückt werden kann, die Menge aller wahren und falschen Sätze; der Umfang dieser Menge hängt aber offensichtlich von unserer Sprache ab, wenn jeder dieser Sätze in unsere Sprache (eine weitere Menge von Sätzen) übersetzbar sein muss; also ist Wahrheit ihrer Extension nach sprachrelativ. Das Bivalenzprinzip als Kriterium des Realismus gilt somit nicht für alle Sätze, sondern maximal für die Sätze, die tatsächlich in einer gegebenen Sprache ausgedrückt werden können. Davidson entpuppt sich - wie bereits erwähnt - als verkappter Antirealist. Wenn aber die vorangegangenen Überlegungen gezeigt haben, dass Davidsons Übersetzbarkeitskriterium falsch sein muss und Sprachlichkeit nicht von Versteh- oder gar Übersetzbarkeit abhängt, dann sollte sich daraus ein schlichtes, aber wirksames Argument zugunsten des Realismus entwickeln lassen:

(1) Wenn unverstehbare Äußerungen möglich sind, dann muss der Wahrheitsbegriff realistisch verstanden werden.

(2) Unverstehbare Äußerungen sind möglich

(3) Also muss Wahrheit realistisch verstanden werden.

Wie lässt sich Prämisse (1) begründen? Wenn partiell oder vollständig uninterpretierbare Sprachen denkbar sind, dann gibt es mindestens eine Äußerung in einer solchen Sprache, die nicht verstanden werden kann. Nun hat eine sprachliche Äußerung eine Bedeutung, sie ist Träger eines propositionalen Gehalts und hat bestimmte Wahrheitsbedingungen und ist daher je nach Lage der Tatsachen wahr oder falsch. Demnach müssen wir, wenn wir die Möglichkeit unverstehbarer Äußerungen zulassen, auch zugestehen, dass diese Äußerungen eine Bedeutung und einen Wahrheitswert haben, auch wenn beides für uns nicht erkennbar ist. Für Davidson sind die Begriffe von Wahrheit und Bedeutung nur sinnvoll anwendbar innerhalb der eigenen Sprache bzw. auf solche Sprachen, die in unsere übersetzbar und damit für uns interpretierbar sind. Wenn es aber unverstehbare, bedeutungsvolle und wahre Sätze gibt, dann ist Wahrheit nicht mehr sprachabhängig und alle Sätze, unabhängig von ihrer möglichen Verstehbarkeit, sind wahr oder falsch, wie es ein realistischer Wahrheitsbegriff fordert. Die Extension des Wahrheitsbegriffs und damit die Gültigkeit des Bivalenzprinzips gehen über den Bereich unserer Sprache hinaus - Wahrheit kann also nicht von unserer Sprache und unserem Begriffsschema abhängen.

Dieses Argument liefert nicht nur einen entscheidenden Grund für den Realismus, sondern verändert auch unser Verständnis dieses Begriffs. Realismus wird üblicherweise in einem negativen Sinn verstanden, d.h. als Ablehnung der These, dass die Wirklichkeit von unserer Sprache oder unserem Denken abhängt. ${ }^{28}$ Leitet man den Realismus jedoch aus der Möglichkeit unverstehbarer Sprachen ab, ergibt sich eine stärkere, positive Variante des Realismus, dass nämlich die Wirklichkeit unsere 
Erkenntnisfähigkeit faktisch übersteigt. Die Denkbarkeit des Unsagbaren ist ein guter Grund für den Realismus, und der Realismus wiederum ist ein guter Grund für die Annahme einer Begrenztheit unserer Erkenntnisfähigkeit. ${ }^{29}$

Nun könnte man einwenden: Es heißt, dass der Antirealismus Wahrheit und Bedeutung von unserer Sprache abhängig macht. Aber wieso sollte es gerade auf unsere Sprache ankommen? Was der Antirealismus behauptet, ist doch nur, dass die Idee einer Wahrheit, die nicht erkannt und nicht ausgedrückt werden kann, in sich widersprüchlich ist - nirgends wurde gesagt, dass wir es sein müssen, für die diese Wahrheit ausdrückbar ist.

Dieser Einwand ist zwar grundsätzlich berechtigt, denn es ist in der Tat ein gravierender Unterschied, ob Wahrheit von unserer Sprache oder von Sprache überhaupt abhängen soll, dürfte aber zu einer für den Antirealisten unerwarteten Konsequenz führen. Denn wenn es die These des Antirealismus ist, dass Wahrheit immer sprachabhängig ist und zwar nur abhängig von irgendeiner logisch möglichen Sprache, löst sich der Unterschied zwischen Realismus und Antirealismus auf. Nicht in dem Sinne, dass beide Positionen sich als konfus und inkohärent herausstellen, sondern dass der Antirealismus nicht mehr vom Realismus unterscheidbar ist. Das wird verständlich, wenn wir einen Blick auf ein ähnliches Problem in Berkeleys Philosophie werfen.

Berkeley behauptet bekanntlich, dass das Sein aller Gegenstände in ihrem Wahrgenommen-Werden besteht (esse est percipi). Die Realität eines Gegenstands ist abhängig davon, dass er Inhalt eines mentalen Zustands ist. Das trägt inm den naheliegenden Vorwurf ein, dass nach seiner Theorie die Dinge der Außenwelt immer vernichtet und neu erschaffen werden, wann immer sie aus dem Bewusstsein heraus- oder in das Bewusstsein hineintreten. Die Welt wäre auf absurde Weise das Produkt unserer Phantasie. Berkeley entgegnet, dass dies keineswegs der Fall sein muss, denn es kommt nicht allein auf unseren Geist an:

For though we hold indeed the objects of sense to be nothing else but ideas which cannot exist unperceived; yet we may not hence conclude they have no existence except only while they are perceived by us, since there may be some other spirit that perceives them, though we do not. Wherever bodies are said to have no existence without the mind, I would not be understood to mean this or that particular mind, but all minds whatsoever. ${ }^{30}$

Es ist nicht unser eigener Geist, von dem für Berkeley die Existenz der Dinge abhängt, sondern nur die Tatsache, Inhalt irgendeines Geistes zu sein. Was von mir nicht wahrgenommen wird, ist deshalb nicht weniger real, solange es nur von einem anderen Geist wahrgenommen wird: „Thus when I shut my eyes, the things I saw may still exist, but it must be in another mind. ${ }^{131}$ Es bleibt allerdings die Frage, was mit Gegenständen ist, die zufällig gerade von niemandem wahrgenommen werden - sind

\footnotetext{
${ }^{29}$ Das hat Rorty in seiner Auseinandersetzung mit Davidson prinzipiell richtig gesehen, auch wenn er die realistische Position dramatischer darstellt als sie eigentlich ist: „What he [der Realist] wants is precisely what the Davidson-Stroud argument prevents him from having - the notion of a world so 'independent of our knowledge' that it might, for all we know, prove to contain none of the things we have always thought we were talking about." (Rorty 1981, 14)

30 Berkeley 1949, 61.

31 Berkeley 1949, 80
} 
sie nicht real? Berkeley bestreitet das. Aus der Geistabhängigkeit aller Gegenstände folgt für inn gerade nicht ihre Irrealität - vielmehr folgt aus der Tatsache, dass sie sowohl geistabhängig als auch real sind, dass es einen unendlichen Geist gibt, der alle Gegenstände wahrnimmt: „Sensible things do really exist: and if they really exist, they are necessarily perceived by an infinite mind. ${ }^{\prime 32}$ Wenn Berkeley behauptet, dass alle Dinge real sind, insofern sie Inhalt eines Bewusstseins sind, so denkt er dabei nicht bloß an finite, menschliche Bewusstseine, sondern auch an ein infinites Bewusstsein, den Geist Gottes. Dann ist ein Berkeley'sches System aber trotz der angenommenen Geistabhängigkeit nicht antirealistisch: Die Wirklichkeit ist unabhängig von unserem Denken, das Bivalenzprinzip und die Evidenztranszendenz der Wahrheit bleiben erhalten, denn jede Tatsache, auch wenn sie uns gegenwärtig nicht bewusst ist, ist Inhalt des infiniten Bewusstseins, so dass für jede Tatsache unabhängig von unserem epistemischen Zugang zu ihr klar bestimmt ist, ob sie besteht oder nicht. ${ }^{33}$

Dieser Gedanke lässt sich nun analog in eine semantische Form bringen und auf Davidsons Antirealismus übertragen: Nehmen wir an, es gäbe ein infinites Bewusstsein, das fähig ist, seine Inhalte sprachlich auszudrücken. Dann sind alle Tatsachen Inhalt dieses infiniten Bewusstseins, da seine Kenntnis der Wirklichkeit durch nichts beschränkt wird. Ein solches Wesen wäre allwissend im Sinne des klassischen Theismus (für jede Tatsache $p$ gilt, dass das Wesen weiß, dass $p$ ). Wenn nun sämtliche Tatsachen Gegenstand des Bewusstseins dieses infiniten Wesens sind und es zugleich fähig ist, die Inhalte seines Bewusstseins sprachlich auszudrücken, dann wird die Sprache dieses Wesens das sein, was ich eine totale Sprache nennen möchte: ${ }^{34}$

Eine Sprache $L_{1}$ ist eine totale Sprache genau dann, wenn für jeden Sachverhalt $p$ gilt: Es existiert ein Satz $S$ in $L_{1}$, der ausdrückt, dass $p .{ }^{35}$

Grundlage ist der Begriff einer möglichen Sprache, den Lewis in Convention entworfen hat. ${ }^{36}$ Demnach ist eine mögliche Sprache $L_{1}$ eine Funktion, die einem beliebigen Satz $\sigma$ aus der Menge aller Sätze in $\mathrm{L}_{1}$ eine Interpretation zuordnet, die aus einem Modus $\mu$ (Indikativ, Imperativ etc.) und einer Wahrheitsbedingung $\tau$ besteht, wobei $\tau$ die Menge aller möglichen Welten ist, für die $\sigma$ in dem durch $\mu$ bestimmten Sinn wahr ist. ${ }^{37}$ Nennen wir die Menge aller Sätze einer Sprache L und die Menge aller Sachverhalte (in allen möglichen Welten) W. Für Sprachen, die keine totalen Sprachen sind, wird dann immer gelten, dass sich nicht alle Elemente aus W eindeutig einem Element aus L zuordnen

\footnotetext{
32 Berkeley 1949, 212.

${ }^{33}$ Devitt 1991, 15 irrt, wenn er Berkeley zum Antirealisten erklärt. Es wäre treffender, von Immaterialismus zu sprechen, denn es ist nicht die unabhängige Realität der Dinge, die in Frage steht, sondern ihre ontologische Beschaffenheit. Zu Recht schreibt daher Dummett 1993, 464 Berkeley einen sophisticated realism zu. Ähnlich auch Mackie 1982, 66.

${ }^{34}$ Nicht zu verwechseln mit dem Begriff einer universalen Sprache, der selbst wiederum verschiedene Deutungen zulässt. (vgl. Loeb 2017, 16ff.)

35 Versteht man eine Sprache als Menge all ihrer Sätze, dann kann es mehr als eine totale Sprache geben. Zwar ist die Welt als die Menge aller Tatsachen immer gleich, aber es gibt immer unendlich verschiedene Repräsentationen der gleichen Tatsache (die Tatsache, dass es regnet, kann durch es regnet, il pleut oder 下雨 ausgedrückt werden). Versteht man eine Sprache als die Menge aller darin ausgedrückten Propositionen, sind alle totalen Sprachen reduzierbar auf eine.

${ }^{36}$ Lewis 1969, $160 \mathrm{ff}$.

37 Lewis 1969, 163.
} 
lassen, d.h. es ist nicht für alle Sätze möglich, ihnen eine Interpretation zuzuordnen $(W>L)$ : Alle Sätze aus $L$ haben eine Wahrheitsbedingung aus W, aber nicht umgekehrt. Der Grund dafür ist, dass wir, um einen Satz zu interpretieren, d.h. um ihm eine Wahrheitsbedingung zuordnen zu können, fähig sein müssen, diese Wahrheitsbedingung zu verstehen. Wir müssen wissen, unter welchen Bedingungen der Satz wahr wäre. Das spricht aber nicht dagegen, dass jeder Satz eine Wahrheitsbedingung hat, denn (wenn wir Lewis' Schema sozusagen rückwärts lesen) jedem Sachverhalt $p$ kann ein Satz $S$ zugeordnet werden, so dass $S$ genau dann wahr ist, wenn $p-$ jeder Sachverhalt kann prinzipiell (sofern es eine entsprechende Funktion gibt, die $\mathrm{p}$ und $\mathrm{S}$ einander korreliert) sprachlich ausgedrückt werden, wenn auch nicht in jeder Sprache. Die faktische Zuordnung von Interpretationen zu Sätzen allerdings unterliegt kontingenten, epistemischen Beschränkungen, nämlich der Fähigkeit eines Subjekts, zu erkennen, dass es der Fall ist, dass p. Eine totale Sprache ist dann der Grenzfall, in dem diese Beschränkungen gleich Null sind. In ihr entspricht jedem Element aus $\mathrm{W}$ ein Element aus $\mathrm{L}(\mathrm{W}=\mathrm{L})$ : Jeder Satz aus $\mathrm{L}$ hat eine Wahrheitsbedingung aus $\mathrm{W}$ und jeder Sachverhalt aus W ist Wahrheitsbedingung mindestens eines Satzes aus L.

Akzeptieren wir dieses Verständnis einer Sprache als Interpretationsfunktion von Ausdrücken, dann sind alle logisch möglichen Sprachen (insbesondere die Sprachen finiter Wesen wie wir) Teilmengen einer totalen Sprache, die sich ganz, teilweise oder gar nicht überschneiden können. Wenn der Antirealismus nur erfordert, dass Wahrheit von einer logisch möglichen Sprache abhängig ist (und nicht von unserer Sprache), dann könnten wir auch annehmen, dass Wahrheit von einer totalen Sprache abhängt: Wahrheit wäre noch immer sprachabhängig, nur eben von der Sprache eines infiniten Wesens. Dann aber verliert die Unterscheidung von Realismus und Antirealismus ihren Sinn. Wenn sie überhaupt einen Sinn haben soll, muss sie die Unabhängigkeit auf finite Wesen beschränken, denn eine Welt, die von einem allwissenden, infiniten Bewusstsein abhängt, unterscheidet sich in nichts von einer Welt, die von nichts als sich selbst abhängt. Und ob Wahrheit sprachrelativ ist oder nicht, macht ebenfalls keinen Unterschied mehr, wenn die einzige Entscheidung die zwischen Unabhängigkeit und Abhängigkeit von einer totalen Sprache ist, denn in beiden Fällen wird die Menge aller wahren Sätze den gleichen Umfang haben, und in beiden Fällen kann es keine Wahrheitswertlücken geben. Wenn ein infinites, allwissendes Wesen denkbar ist, das über eine totale Sprache verfügt, dann folgt bereits aus dem Begriff der totalen Sprache und der Allwissenheit, dass es notwendigerweise keine Wahrheit gibt, die dieses Wesen nicht ausdrücken kann, ganz egal, ob der Wahrheitsbegriff bivalent verstanden wird oder nicht. Natürlich blieben alle Wahrheiten abhängig von der totalen Sprache des infiniten Wesens, denn Wahrheit als Eigenschaft bestimmter Propositionen besteht nach antirealistischem Verständnis nur dann, wenn es ein Subjekt gibt, das diese Propositionen glaubt. Aber ein allwissendes Wesen glaubt alle wahren Propositionen und nur wahre Propositionen, auch wenn die Wahrheit dieser Propositionen nicht daraus resultiert, 
dass sie geglaubt werden, sondern beides - Wahrheit und Glauben - koinzidiert. ${ }^{38}$ Daher verschwindet der Unterschied zwischen dem, was wahr ist, und dem, was prinzipiell für wahr gehalten werden kann, und auch das Konzept der Evidenztranszendenz verliert seinen Sinn. Denn Wahrheit und Evidenz fallen für das infinite Bewusstsein in eins, was freilich nicht so zu verstehen ist, dass Wahrheit an Erkennbarkeit geknüpft ist - es gibt schlicht keinen Unterschied mehr zwischen Wahrheit und Erkennbarkeit. Das Bivalenzprinzip hingegen, das das eigentlich relevante Kriterium des Realismus ist und aus dem sich nur unter der Annahme, dass wir von finiten Wesen sprechen, die Evidenztranszendenz als zweites Merkmal des Realismus ergibt, kann aufrechterhalten werden. Akzeptiert man also den ursprünglichen Einwand, dann hat sich die antirealistische Position aufgelöst. Es gibt nichts mehr, worin sich der Antirealismus als Abhängigkeit von (ontologisch) einem infiniten Wesen oder (semantisch) einer totalen Sprache vom Realismus unterscheiden würde.

In Wittgensteins Zetteln findet sich eine Notiz zum Konflikt zwischen Realismus und Antirealismus:

\begin{abstract}
Einer sei ein überzeugter Realist, der Andere ein überzeugter Idealist und lehrt seine Kinder dementsprechend. In einer so wichtigen Sache, wie der Existenz, oder Nichtexistenz der äußern Welt wollen sie ihren Kindern nichts Falsches beibringen. [...] Aber der Idealist wird den Kindern doch das Wort ,Sessel' beibringen, denn er will sie ja lehren, dies und jenes zu tun, z.B. einen Sessel zu holen. Wo wird sich also, was die idealistisch erzogenen Kinder sagen, von dem, was die realistischen sagen, unterscheiden? Wird der Unterschied nicht nur einer der Schlachtrufe sein ${ }^{39}$
\end{abstract}

Die Annahme eines infiniten Wesens oder einer totalen Sprache als Bezugspunkt der Abhängigkeitsrelation läuft genau auf das hinaus, was Wittgenstein hier beschreibt - an keiner Stelle ist ein signifikanter Unterschied feststellbar. Antirealismus ist Realismus. Und während in der ontologischen Deutung die Entscheidung zwischen Realismus und Antirealismus noch eine interessante Konsequenz hat - nämlich die Frage, ob es ein infinites Bewusstsein gibt, von dem die Welt abhängt, oder nicht - fällt mit der semantischen Deutung selbst dieser Aspekt weg. Denn es ist nicht notwendig, dass eine totale Sprache tatsächlich realisiert ist. Wenn Antirealismus nicht mehr als die prinzipielle Abhängigkeit des Realen von einer möglichen Sprache bedeuten soll, dann genügt

\footnotetext{
${ }^{38} \mathrm{vgl}$. Plantinga 1982, 69.

39 Wittgenstein, Zettel $413 \mathrm{f}$.
} 
allein die Denkbarkeit einer totalen Sprache, ${ }^{40}$ um zu zeigen, dass der Unterschied zwischen

Realismus und Antirealismus letztlich fiktiv ist. Beide sind in letzter Konsequenz identisch. ${ }^{41}$

Literatur:

Berkeley, George 1949: The Works of George Berkeley, Bishop of Cloyne. Bd. 2: The Principles of Human Knowledge. First Draft of the Introduction to the Principles. Three Dialogues between Hylas and Philonous. Philosophical Correspondence with Johnson, London.

Davidson, Donald 1974: On the very idea of a conceptual scheme, in: Proceedings and Addresses of the American Philosophical Association 47, 5-20.

Dennett, Daniel 1981: Brainstorms. Philosophical essays on mind and psychology, Cambridge, MA.

Devitt, Michael 1991: Realism and Truth, Oxford.

Dummett, Michael 1993: The seas of language, Oxford.

Husserl, Edmund 1950: Cartesianische Meditationen und Pariser Vorträge. (Hua 1), Den Haag.

Lewis, David 1969: Convention, Harvard.

Loeb, Iris 2017: The role of universal language in the early work of Carnap and Tarski, in: Synthese $194,15-31$.

Mackie, John Leslie 1982: The miracle of theism. Arguments for and against the existence of God, Oxford.

Nagel, Thomas 1986: The View from Nowhere, Oxford.

Plantinga, Alvin 1982: How to be an anti-realist, in: Proceedings and Addresses of the American Philosophical Association 56, 47-70.

Putnam, Hilary 1981: Reason, Truth and History, Cambridge.

Rescher, Nicholas 1980: Conceptual schemes, in: Midwest Studies in Philosophy 5, 323-346.

Rorty, Richard 1981: Consequences of Pragmatism, Brighton.

Sankey, Howard 1990: In defence of untranslatability, in: Australasian Journal of Philosophy 68, 1-21.

Snell, Bruno 1975: Die Entdeckung des Geistes, Göttingen

\footnotetext{
${ }^{40}$ Man könnte einwenden, dass eine totale Sprache nicht möglich ist, da eine solche Sprache semantisch geschlossen sein müsste (sie enthält ihr eigenes Wahrheitsprädikat und selbstreferentielle Ausdrücke) und semantisch geschlossene Sprachen nach Tarski inkonsistent sind (Tarski 1935, 278f.). Die Inkonsistenz derartiger Sprachen bedeutet für Tarski jedoch nicht, dass der Begriff einer semantisch geschlossenen Sprache inkonsistent ist, sondern, dass sie notwendigerweise Widersprüche implizieren wie z.B. das Lügner-Paradox. Das gilt z.B. für die natürlichen Sprachen. Aber aus der Tatsache, dass natürliche Sprachen Paradoxien implizieren, folgt nicht, dass der Begriff der natürlichen Sprache inkonsistent ist oder es gar keine natürlichen Sprachen geben kann, sondern nur, dass natürliche Sprachen nicht formalisiert werden können, ohne dass Widersprüche ableitbar sind. Es folgt also auch nicht, dass eine totale Sprache unmöglich ist, sondern nur, dass in einer totalen Sprache (vielleicht notwendigerweise) Paradoxien ableitbar sind. Das aber spricht ebenso wenig gegen ihre Denkbarkeit wie die Tatsache, dass natürliche Sprachen Paradoxien enthalten, gegen deren offensichtliche Existenz spricht. Wenn natürliche Sprachen denkbar sind, dann auch eine totale Sprache. (Diesen Einwand verdanke ich einem/r anonymen Gutachter/in.)

${ }^{41}$ Teile dieser Arbeit wurden auf dem Ludwig-Fleck-Kolloquium an der Universität Zürich 2016 vorgetragen, wofür ich Hartmut von Sass zu großem Dank verpflichtet bin.
} 
Willaschek, Marcus 2003: Der mentale Zugang zur Welt. Realismus, Skeptizismus und Intentionalität, Frankfurt/Main.

Wittgenstein, Ludwig 1967: Zettel, Oxford.

Wittgenstein, Ludwig 1984: Tractatus logico-philosophicus. Werkausgabe Bd. 1: Tractatus logicophilosophicus. Tagebücher 1914-1916. Philosophische Untersuchungen, Frankfurt/Main. 